\title{
Una aproximación al significado del amor en una población infantil en Lima, Perú
}

\author{
José Ventura-León; Andy Sánchez-Villena; Miguel Barboza-Palomino; Karen Curahua-Guillén; Laura Oros
}

Cómo citar este artículo:

Ventura-León, J., Sánchez-Villena, A., Barboza-Palomino, M., Curahua-Guillén, K., \& Oros, L. (2022). Una aproximación al significado del amor en una población infantil en Lima, Perú. Acta Colombiana de Psicología, 25(1), 56-71. https:// www.doi.org/10.14718/ACP.2022.25.1.5

Recibido, diciembre 22/2020; Concepto de evaluación, agosto 12/2021; Aceptado, octubre 15/2021

José Ventura-León'
ORCID: https://orcid.org/0000-0003-2996-4244
Universidad Privada del Norte, Lima, Perú.
Andy Sánchez-Villena
ORCID: https://orcid.org/0000-0001-7828-5884
Universidad Privada del Norte, Lima, Perú.
Miguel Barboza-Palomino
ORCID: https://orcid.org/0000-0001-8045-5491
Universidad Privada del Norte, Lima, Perú.
Karen Curahua-Guillén
ORCID: https://orcid.org/0000-0002-6730-0142
Universidad Privada del Norte, Lima, Perú.
Laura Oros
ORCID: https://orcid.org/0000-0003-1265-7651
Universidad Católica Argentina, Buenos Aires, Argentina.
Consejo Nacional de Investigaciones Científicas y Técnicas (CONICET), Buenos Aires, Argentina.
Universidad Adventista del Plata, Entre Ríos, Argentina.

\section{Resumen}

El presente estudio tuvo como objetivo aproximarse al significado del amor, a través de su expresión escrita, en una muestra de niños y niñas de Lima Metropolitana de Perú, y examinar sus diferencias según sexo y edad. En total, se contó con la participación de 289 individuos divididos en dos muestras: una muestra exploratoria $(n=50)$ y otra de confirmación $(n=239)$. El estudio siguió una metodología mixta, a partir del análisis temático como técnica cualitativa para identificar códigos subyacentes a la definición de amor de los niños, y del análisis clúster jerárquico como técnica cuantitativa para generar un dendrograma. Los resultados revelaron la presencia de seis categorías centrales: relación con otros significativos cercanos, romanticismo y amor propio, relación característica entre adultos, estado positivo, afectividad, y apoyo incondicional. A partir de ello, se concluye que el concepto de amor durante la niñez integra tres categorías del esquema interpretativo de las emociones: contextual o elicitador, experiencia subjetiva, y tendencia expresiva/motivacional.

Palabras clave: amor, infantil, significado, estudio mixto.

\footnotetext{
${ }^{1}$ Av. Alfredo Mendiola 6062, Los Olivos 15306, Universidad Privada del Norte, Lima, Perú. Tel.: (511) 6143311. jose.ventura@upn.pe
} 


\title{
An approach to the meaning of love in a child population in Lima, Peru
}

\begin{abstract}
The aim of the present study was to approach the meaning of love, through its written expression, in a sample of boys and girls from Metropolitan Lima, Peru, and to examine its differences according to sex and age. A total of 289 children participated in the study, divided into two samples: an exploratory sample $(n=50)$ and a confirmatory sample $(n=239)$. The study followed a mixed methodology, using thematic analysis as a qualitative technique to identify codes underlying the children's definition of love, and hierarchical cluster analysis as a quantitative technique to generate a dendrogram. The results revealed the presence of six central categories: relationship with significant others, romanticism and self-love, characteristic relationship between adults, positive state, affectivity, and unconditional support. From this, it is concluded that the concept of love during childhood integrates three categories of the interpretative scheme of emotions: contextual or elicitor, subjective experience, and expressive/motivational tendency.

Keywords: love, child, meaning, mixed study.
\end{abstract}

\section{Introducción}

La experiencia de sentirse amado es vital para el desarrollo saludable de los niños (Phillips \& Shonkoff, 2000; Sroufe, 2005). Según las teorías de apego (Bowlby, 1989), es típicamente en la relación entre padres e hijos (Sabey et al., 2018) donde se establecen los lazos afectivos que fomentan la necesidad de ser amado (Melgosa \& Melgosa, 2006), relación que incluso afecta las primeras relaciones románticas de los hijos en la vida adulta (Sabelnikova et al., 2020). Por ende, se puede afirmar que el entorno familiar y social juega un papel importante en la conducta de sus integrantes (Thompson, 2008).

$\mathrm{Al}$ respecto, un estudio revela que familiares próximos - como los abuelos - son vistos como figuras de sostén, modelos de identificación, amor incondicional, cuidado y fuente de consejos (Hess et al., 2020), y que los hermanos son personas queridas, considerados como fuente de intimidady compañía (Facio \& Resett, 2007). Asimismo, se ha encontrado que los amigos también ocupan un lugar importante, porque dejan recuerdos duraderos en el desarrollo del niño (Mallet, 2016). De este modo, se podría concluir que el afecto familiar y amical juegan un papel fundamental en la creación de lazos afectivos (Oros et al., 2015). Sin embargo, las investigaciones acerca del afecto infantil han girado en torno al cuidado (Aslanian, 2015), y han dejado de lado la comprensión de su significado, lo que convierte al amor en la infancia en un asunto poco explorado (Cannoni \& Bombi, 2016).

Por su connotación polisémica, resulta difícil definir el amor (Berscheid, 2010), sin embargo, algunos autores se han aproximado a su definición, indicando que se trata de un sentimiento de unión hacia alguien o algo (Lucas et al., 2019), una emoción básica (Shaver et al., 1996), un vínculo (Harlow, 1958), o una acción (Chabot, 2008; Fromm, 2004; Rubin, 1970; Scott, 1997) que conlleva a la transformación del individuo (Aslanian, 2015) y al bienestar psicológico (Oros \& Richaud, 2011). Asimismo, se han encontrado clasificaciones del amor, en las que se pueden encontrar el hecho romántico (Bartels \& Zeki, 2000; Rubin, 1970) o compasivo (Berscheid, 2010), o un acto de compañerismo (Grote \& Frieze, 1994) que surge de la proximidad y conexión interpersonal de una relación segura y positiva (Cavanaugh et al., 2015; Fredrickson, 2013), que cuando se torna desfavorable o no correspondido suele estar acompañado de inseguridad, incertidumbre y angustia (Lazarus, 2000).

Ahora bien, aunque el significado en específico del amor infantil ha sido poco investigado, se suelen encontrar estudios heteronormativos que sugieren que los niños definen el amor como sinónimo de amistad - jugar o hacer algo juntos-, y que abrazarse o besarse y participar en conductas de ayuda (Klein, 1989) podría entenderse de 
manera diferente si estas conductas son dirigidas hacia un compañero del mismo sexo, pues tendrían una connotación amical, o si se dirigen hacia el sexo opuesto, lo que denotaría romanticismo (Cannoni \& Bombi, 2016).

También, en investigaciones en otros contextos culturales, se ha encontrado que el amor en la infancia puede ser entendido como un estado afectivo que experimenta el niño hacia otra persona - un agente externo-(Brechet, 2015), o como un fenómeno cambiante que provoca que el niño se sienta bien cuando le aman, mientras que, si le hacen sentir tristeza o enojo, ya no (Widen \& Russell, 2005). Además, en el marco de las emociones, el amor puede asumirse como un ente subjetivo, expresivo o motivacional (Frijda et al., 2000; Haidt, 2003).

En lo que respecta a las diferencias por sexo, un estudio realizado en el sur y centro de Italia demuestra que las niñas manifiestan mayor interés por el amor romántico, aunque lo expresan menos, mientras que a los niños les interesa más un amor de tipo amical (Cannoni \& Bombi, 2016). Respecto a la expresión romántica, cabe destacar que, durante la niñez, esta tiene una connotación de un vínculo idealizado, en muchos casos fantasioso y no recíproco, que implica atracción por la apariencia física del otro y preferencia por su compañía (Carlson \& Rose, 2007).

Respecto a la edad, algunos autores sostienen que el amor romántico tiende a incrementarse entre los $10 \mathrm{y}$ los 14 años, pero este crecimiento es ondulante, porque presenta subidas y bajadas (Connolly et al., 2004); y otros autores explican que el decrecimiento ocurre a causa de una relación no recíproca (Carlson \& Rose, 2007). Pese a ello, son escasos los estudios de diferencias del amor según la edad, y solo se encuentran aportes investigativos en poblaciones cercanas - adolescentes - que indican que, a mayor edad, mayores son las interacciones sociales diarias y las relaciones con personas cercanas que involucran el aspecto romántico (Adams et al., 2001). Del mismo modo, la percepción del apoyo por parte de una amistad romántica - entendida como una relación cercana y afectuosa entre amigos - tiende incrementarse entre los 9 y los 12 años (Furman \& Buhrmester, 1992).

Por otra parte, algunos estudios demuestran que el no sentirse amado tiene implicancias en diferentes esferas de la vida del niño. Por ejemplo, desde una perspectiva biológica, Harvey et al. (2019) demuestran que la evitación del apego materno tiene un efecto en la pendiente de cortisol diurna; es decir, que una mayor evitación del apego materno predice una pendiente de cortisol diurna más plana, lo que implica problemas de salud. Incluso, Anderson et al. (2018) señalan que la falta de cuidado materno se asocia con una mayor circunferencia de la cintura, altos niveles de insulina y menor distensibilidad arterial; y Bernard et al. (2019) señalan que la calidad de apego en niños en servicio de protección infantil predice significativamente la cantidad de proteína Creactiva ( $\mathrm{PCR})$ - niveles altos de esta proteína denotan inflamación en el cuerpo-

Desde una perspectiva psicológica, McElwain et al. (2015) demuestran que la interacción madre-hijo correspondiente a hacer sentir al niño que es digno de atención, $\mathrm{y}$ saber que otros estarán cuando lo requiera, lo cual predice que el niño efectúe acciones directas para modificar la situación problemática y reduzca las consecuencias negativas. De hecho, en un conjunto de observaciones de juego, se observó en preescolares mayor empatía y relaciones más profundas y mutuas cuando existían estos comportamientos de afecto ejercidos por parte de la madre (Sroufe, 2005). Incluso, un metaanálisis sobre el tema revela que estos comportamientos se encuentran moderadamente relacionados con la comprensión de las emociones, y que esto ayuda a interpretar mejor el comportamiento de otros y responder de manera correcta en situaciones sociales (Cooke et al., 2016). Asimismo, Zimmer-Gembeck et al. (2017) afirman que cuando el padre no responde adecuadamente, o es inconsistente respecto a las demandas afectivas del niño, se evidencia una moderada asociación con que el niño espere que suceda lo peor.

Partiendo de lo anterior, el presente estudio se justifica desde tres aspectos: (a) desde lo teórico, porque busca incrementar el marco conceptual, cuya literatura científica en español es escasa, de manera que resulta oportuno estructurar un estudio - de carácter fundamentalmente exploratorioque permita dar paso a más investigaciones al respecto; (b) en lo práctico, porque intenta conocer el significado que los niños y niñas atribuyen a un término abstracto como el amor, lo cual ayuda en gran medida a actuar desde la educación parental para suministrar acciones encaminadas a activar dicho significado; y (c) en lo social, porque busca 
visibilizar una problemática que entraña factores culturales y puede revelar que la formación del concepto del amor se construye desde contextos próximos como la familia.

En este sentido, el presente estudio tuvo como objetivos: (a) a través de su expresión escrita, aproximarse al significado del amor en una muestra de niños y niñas de 8 a 12 años - los rangos de edades de los niños del presente estudio se encuentran en la denominación "niñez media" (Halfon et al., 2018; Papalia \& Martorell, 2017) — de la ciudad de Lima Metropolitana de Perú; y (b) examinar si existen diferencias en las definiciones según el sexo y la edad de los participantes.

\section{Método}

\section{Tipo de investigación}

Se realizó una investigación mixta, que combina una técnica cualitativa - un análisis temático- y una cuantitativa — un análisis de agrupación jerárquica — para la comprensión de un fenómeno (Johnson \& Onwuegbuzie, 2004), entendiendo el criterio de complementariedad de ambas técnicas (Powell et al., 2008).

\section{Participantes}

Se contó con la participación de 289 niños (45.86 \%) y niñas $(53.79 \%)$ de dos escuelas de Lima Metropolitana, Perú, con edades entre los 8 y los 12 años $(M=9.91$; $D T=1.15)$. Los participantes fueron seleccionados de forma intencional y divididos en dos muestras: una conformada por 50 niños (48 \%) y niñas (52\%) de 8 a 12 años $(M=9.96$; $D T=1.14)$, que constituyeron una muestra exploratoria para elaborar un diccionario de códigos; y otra conformada por 239 niños (45.60\%) y niñas (54.39\%) de 8 a 12 años $(M=9.90 ; D T=1.15)$, con la cual se establecieron los análisis finales del estudio.

La variable edad fue recategorizada en (a) niños y niñas de 8 a 9 años $(n=90)$ y (b) niñas y niños de 10 a 12 años $(n=149)$, debido a que existe evidencia de que el funcionamiento cognitivo relacionado con la planificación, lógica y memoria son diferentes en niños de 10 a 12 años (van der Molen \& Molenaar, 1994), y porque esta diferenciación favorece la comparación por el hecho de que se puede contar con grupos relativamente representativos en cantidades.
Finalmente, cabe mencionar que todos los niños se encontraban entre el tercer y el sexto grado de escolarización primaria, lo cual se tuvo en cuenta con el fin de garantizar que todos los participantes tuvieran adquiridas las habilidades de escritura necesarias para la aplicación del material de investigación.

\section{Instrumento}

Se elaboró una ficha de recolección de datos con dos secciones: en la primera se solicitaron los datos sociodemográficos —edad, grado escolar y nombre de la institución educativa - del participante, y en la segunda se incluyó la pregunta “¿Qué es el amor?” y un espacio de 16 renglones para escribir una respuesta. Se invitó a los niños a escribir en dichos reglones, y se les indicó que no sucedía nada si no completaban todas las líneas, que lo importante era que respondieran a la pregunta formulada. Este método de recolección de información es coherente con estudios previos interesados en el significado de un fenómeno (véase, p. ej., Davis et al., 1998; Lichtenthal et al., 2010).

\section{Procedimiento}

Inicialmente, el proyecto fue aprobado por el Comité de Ética de la Universidad Privada del Norte (UPN) de Perú. Tras esta aprobación, se solicitaron los permisos y se coordinó con dos escuelas para la aplicación de la ficha de recolección. La primera escuela fue considerada como una muestra exploratoria $(n=50)$ y la segunda escuela como una muestra de confirmación $(n=239)$. En ambos momentos, los padres firmaron un consentimiento informado en el que se explicaron los fines de la investigación, el uso de la información y la participación voluntaria. La aplicación fue realizada por una psicóloga y un asistente de investigación debidamente capacitado, quienes leyeron las instrucciones con la finalidad de estandarizar el proceso. La aplicación no contaba con un tiempo límite, pero tomó en promedio 15 minutos. Dicha aplicación se realizó en el último trimestre del 2019, de forma colectiva dentro del aula, y en el horario habitual de clases.

Se establecieron dos momentos de análisis: uno exploratorio, o piloto, $(n=50)$ donde se recolectó información de una escuela de nivel socioeconómico medio con el fin elaborar un diccionario de códigos asociados al significado del amor — que no guardaban relación de orden entre sí y 
eran mutuamente excluyentes- (Olabuénaga \& Ispizua, 1989), para lo cual se utilizó una técnica cualitativa —el análisis temático-, siguiendo las recomendaciones de Braun y Clarke (2006); y un segundo momento, con una muestra final, o confirmatoria, $(n=239)$ donde se aplicó el mismo protocolo de evaluación a una nueva escuela - con características sociodemográficas similares - con el fin de observar la presencia de los códigos establecidos en la muestra piloto - se asignó un 1 ante la presencia del código y un 0 si no lo había-, un procedimiento realizado por un investigador diferente a quien elaboró el diccionario de códigos en la muestra piloto.

\section{Análisis de datos}

Inicialmente, el análisis de datos siguió una lógica cualitativa, para la cual las respuestas de los niños fueron transcritas de forma literal en una hoja de cálculo de Excel®; luego, un investigador realizó el análisis temático de acuerdo con las recomendaciones de Braun y Clarke (2006): (a) familiarización con las respuestas, lo que implicó la lectura y relectura de los datos recolectados, para así reconocer las respuestas de los participantes e ir construyendo algunas interpretaciones y códigos; (b) segmentación, donde se identificó y seleccionó segmentos de información relevantes para responder a la pregunta de investigación; (c) elaboración de un diccionario de códigos (véase Tabla 1), con base en la familiarización de los datos y la revisión de la literatura; (d) codificación, que consistió en la asignación de códigos - conceptos — a cada segmento de información seleccionado; y (e) vinculación, donde se mantuvieron los códigos vinculados en más de la mitad del total de casos analizados.

En un segundo momento, se siguió una lógica cuantitativa con una nueva muestra, donde se verificó la presencia de los códigos previamente establecidos — se asignó la puntuación de 1 o 0 según los criterios antes descritos-. En este punto, se calculó una matriz de correlaciones con los códigos mediante el software R, versión 4.0.2, (R Core Team, 2020), a partir de la propuesta de Liu et al. (2012); y se utilizó el análisis cluster a través de la librería factoextra (Kassambara \& Mundt, 2020). Específicamente, se empleó el análisis clúster jerárquico con el método de agrupación aglomerativo, en donde cada elemento inicia con un grupo separado y luego se fusiona en un grupo cada vez más grande; este paso se repite hasta formar un número de grupos determinados, y el resultado es un diagrama en forma de árbol, denominado dendrograma (Gan et al., 2007), que permite examinar la similitud de los códigos. Este gráfico es de gran utilidad porque permite establecer una taxonomía respecto a cómo se organiza el significado del amor en la población infantil, y así favorecer su interpretación. Previo a este análisis, se determinó el número de clúster mediante el método silhouette, por ser considerado el más apropiado (Rousseeuw, 1987). Finalmente, estos resultados fueron analizados por los investigadores para obtener subcategorías, interpretadas con base en la literatura científica.

Por último, se calcularon frecuencias relativas para cada categoría de respuesta en la muestra total y según el sexo y la edad de los participantes; y para establecer la magnitud de la diferencia entre las proporciones se utilizó el coeficiente $h$ de Cohen, donde un $h \geq 0.20$ significa un tamaño de efecto pequeño, un $h \geq 0.50$, un tamaño de efecto mediano, y un $h \geq 0.80$, un tamaño de efecto grande (Cohen, 1988).

\section{Resultados}

\section{Fase cualitativa}

En la Tabla 1 se presentan los resultados de la muestra piloto $(n=50)$ que surgieron de la codificación con base en el análisis temático. Los resultados demuestran la existencia de 16 códigos nominales mutuamente excluyentes, que no guardan relación de orden entre sí.

\section{Fase cuantitativa}

En la Tabla 2 se presentan los estadísticos descriptivos de la codificación realizada por el evaluador. Se observa que, en total, las categorías que más aparecen son alguien/ enamorado, padres y familia, mientras que las menos frecuentes son compañía y expresión material. En cuanto al sexo, se observa que las niñas presentan mayores tasas de frecuencia en las categorías alguien, familia, amigos, respeto, incondicionalidad, felicidad, bienestar y corporalidad, en comparación con los niños. En el resto de las categorías son los niños quienes superan a las niñas, aunque el coeficiente $h$ revela pequeñas diferencias en sus proporciones. Respecto a la edad, se observa que los niños y niñas de entre 8 y 9 años presentaron una mayor tasa de frecuencia en las categorías alguien, amigos, relación con otros, respeto, felicidad, expresión material, corporalidad y visión negativa. 


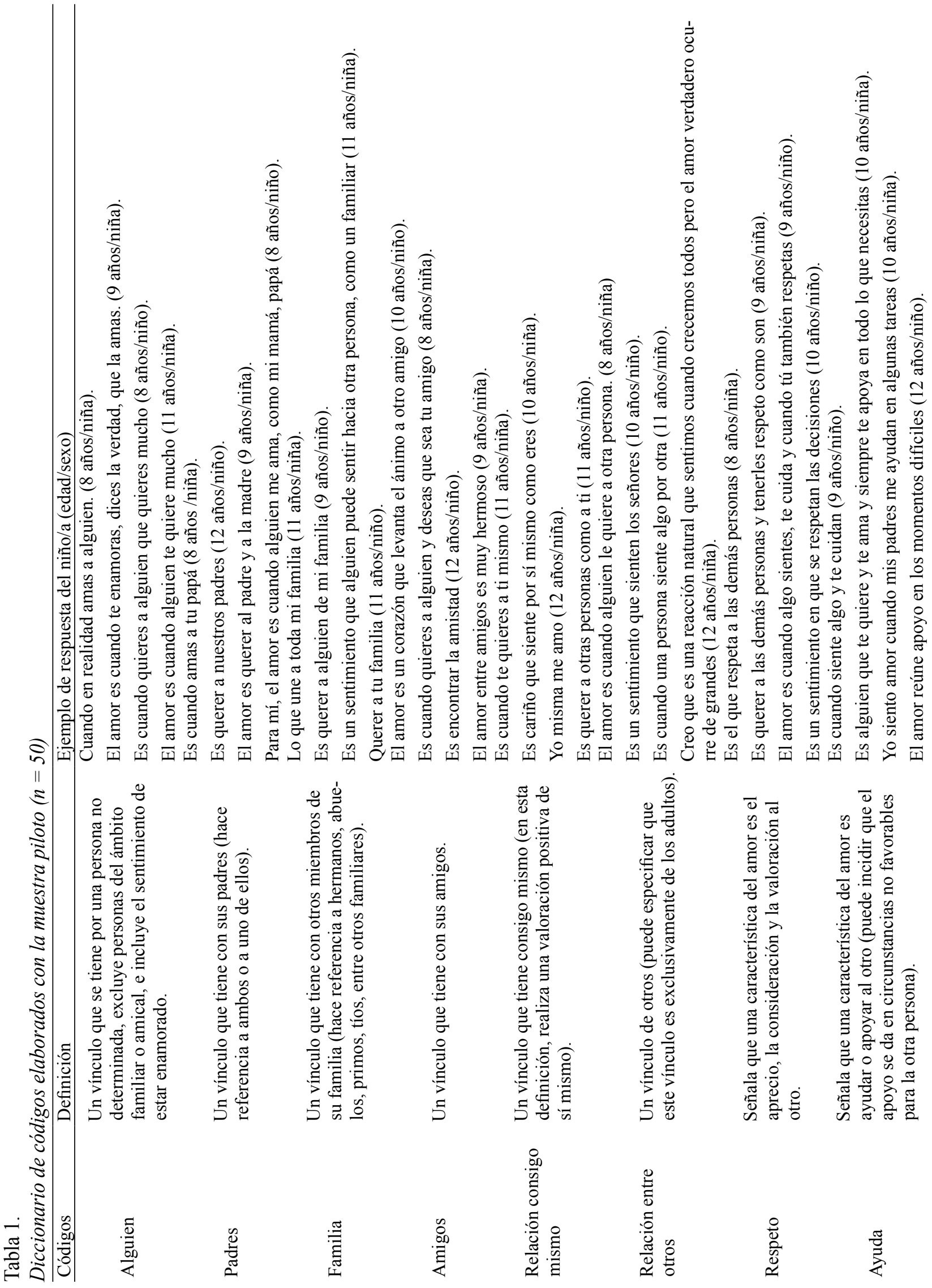




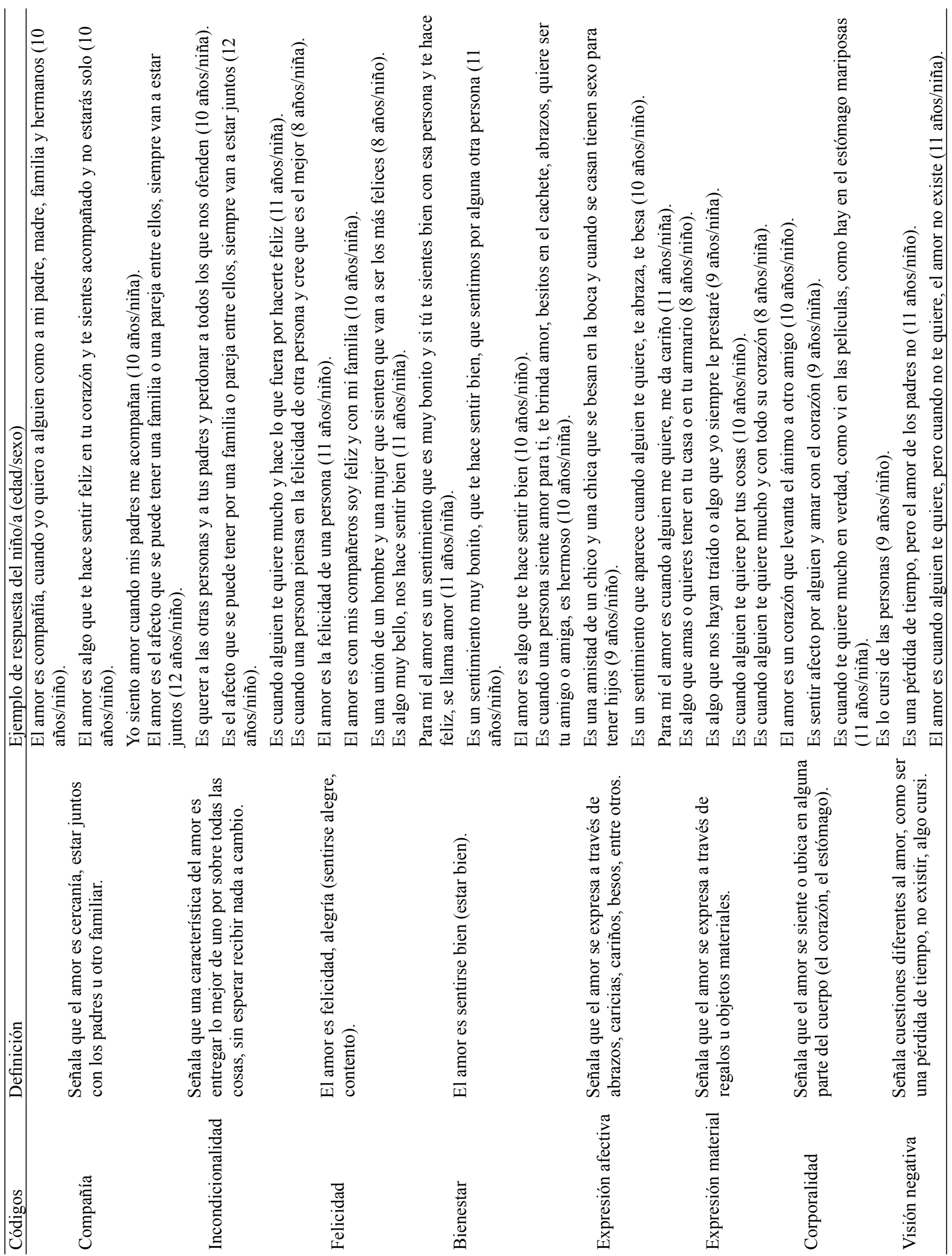


Tabla 2.

Estadísticos descriptivos de las categorías del significado del amor infantil $(n=239)$

\begin{tabular}{|c|c|c|c|c|c|c|c|}
\hline \multirow{2}{*}{ Categorías } & \multirow{2}{*}{$\%$ total } & \multicolumn{3}{|c|}{$\%$ sexo } & \multicolumn{3}{|c|}{$\%$ edad } \\
\hline & & Niñas & Niños & $h$ & 8 a 9 años & 10 a 12 años & $h$ \\
\hline Alguien & 49 & 55 & 43 & 0.24 & 62 & 42 & 0.40 \\
\hline Padres & 18 & 18 & 19 & 0.03 & 12 & 22 & 0.27 \\
\hline Familia & 18 & 22 & 12 & 0.27 & 9 & 23 & 0.39 \\
\hline Amigos & 16 & 19 & 13 & 0.16 & 18 & 15 & 0.08 \\
\hline Relación consigo mismo & 4 & 4 & 4 & 0.00 & 2 & 5 & 0.17 \\
\hline Relación con otros & 15 & 12 & 17 & 0.14 & 17 & 13 & 0.11 \\
\hline Respeto & 5 & 8 & 2 & 0.29 & 7 & 4 & 0.13 \\
\hline Ayuda & 6 & 3 & 9 & 0.26 & 4 & 7 & 0.13 \\
\hline Compañía & 1 & 1 & 2 & 0.08 & 0 & 2 & 0.28 \\
\hline Incondicionalidad & 3 & 5 & 1 & 0.25 & 0 & 5 & 0.45 \\
\hline Felicidad & 10 & 11 & 8 & 0.10 & 11 & 9 & 0.07 \\
\hline Bienestar & 7 & 8 & 6 & 0.08 & 4 & 9 & 0.21 \\
\hline Expresión afectiva & 5 & 5 & 5 & 0.00 & 6 & 5 & 0.04 \\
\hline Expresión material & 1 & 1 & 2 & 0.08 & 2 & 1 & 0.08 \\
\hline Corporalidad & 5 & 7 & 2 & 0.25 & 6 & 4 & 0.09 \\
\hline Visión negativa & 3 & 2 & 5 & 0.17 & 4 & 3 & 0.05 \\
\hline
\end{tabular}

Nota. \%: cantidad de veces en porcentaje que fue detectada la categoría por el evaluador en la población total, según sexo y edad. $h: h$ de Cohen (tamaño del efecto).

En el resto de las categorías, los niños y niñas de 10 a 12 años muestran mayores porcentajes, pero el coeficiente $h$ informa pequeñas diferencias en las proporciones en todas las categorías, a excepción de incondicionalidad, que se acerca a un efecto mediano $(h=0.45)$.

En la Figura 1 se presenta un dendrograma con la información de una matriz de correlación, calculada mediante el análisis de los códigos. Los resultados revelan la presencia de seis clúster o subcategorías. El primero es denominado significativos cercanos - padres, familia, amigos - , y consiste en el vínculo que tiene el niño con sus progenitores, otros miembros de su familia, y sus amigos. El segundo, afectividad — expresión afectiva, respeto, visión negativa, relación con otros-, hace alusión a la expresión a través de abrazos, caricias, cariños, besos y valoración del otro, que puede experimentarse como algo no placentero. El tercero, romanticismo — corporalidad y alguien —, es el afecto por una persona cercana no especificada por el menor, y que proviene del corazón o produce sensaciones corporales —estómago - El cuarto, centrado en sí mismo — expresión material y relación consigo-, consiste en expresar aprecio por uno mismo a través de la entrega o pertenencia de un objeto material. El quinto, estado positivo - felicidad, bienestar-, involucra la consideración del amor como un estado de alegría y felicidad que genera bienestar. Y sexto, apoyo incondicional -incondicionalidad, ayuda, compañía-, implica ayudar a alguien entregando lo mejor de uno por sobre todas las cosas, estando próximo en momentos de dificultad, sin esperar recibir nada a cambio.

En lo que respecta al sexo de los participantes, se observa una estructura similar en niños y niñas; sin embargo, ambos dendrogramas tienen variaciones respecto al modelo general (véase Figura 2). En este sentido, se evidencia la desestructuración de la subcategoría romanticismo en los niños, pues los códigos alguien y corporalidad se dispersan a otras subcategorías. En el caso de la afectividad, se evidenció una división de los códigos que dio lugar a los denominados afectividad negativa y afectividad positiva. Asimismo, la subcategoría incondicionalidad está compuesta por compañia en niñas y corporalidad en niños. Un caso particular es la subcategoría centrado en sí mismo, que en las niñas involucra expresión afectiva, relación consigo mismo y respeto, mientras que en los niños es relación consigo mismo, alguien y expresión material. Finalmente, el código compañia pertenece a la subcategoría estado positivo en los niños.

En cuanto a la edad, existen diferencias sustanciales entre niños de 8 a 9 años y de 10 a 12 años, sin embargo, 
Figura 1.

Dendrograma de los códigos, subcategorías y categorías

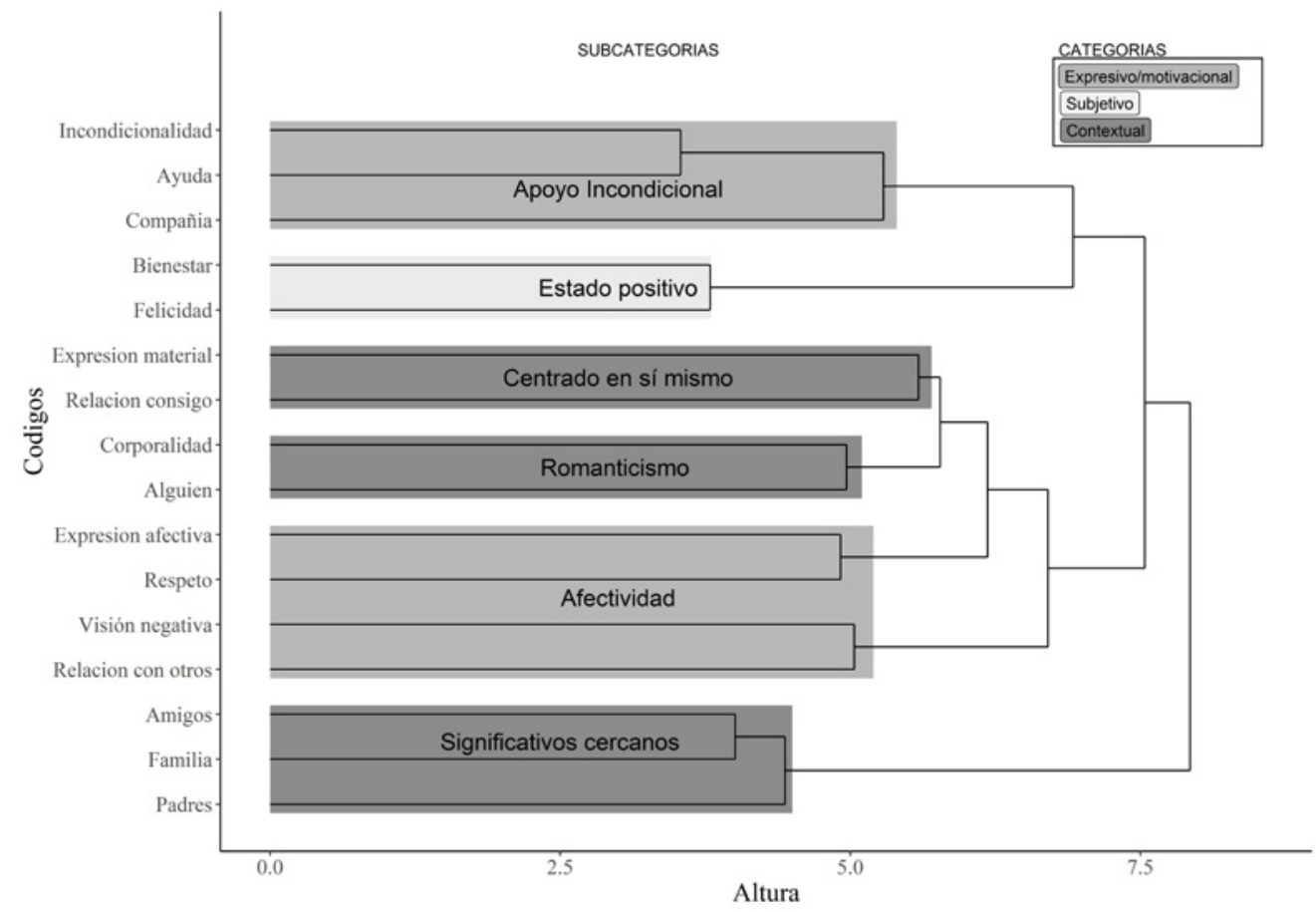

Figura 2.

Dendrograma de los códigos, subcategorías y categorías según el sexo
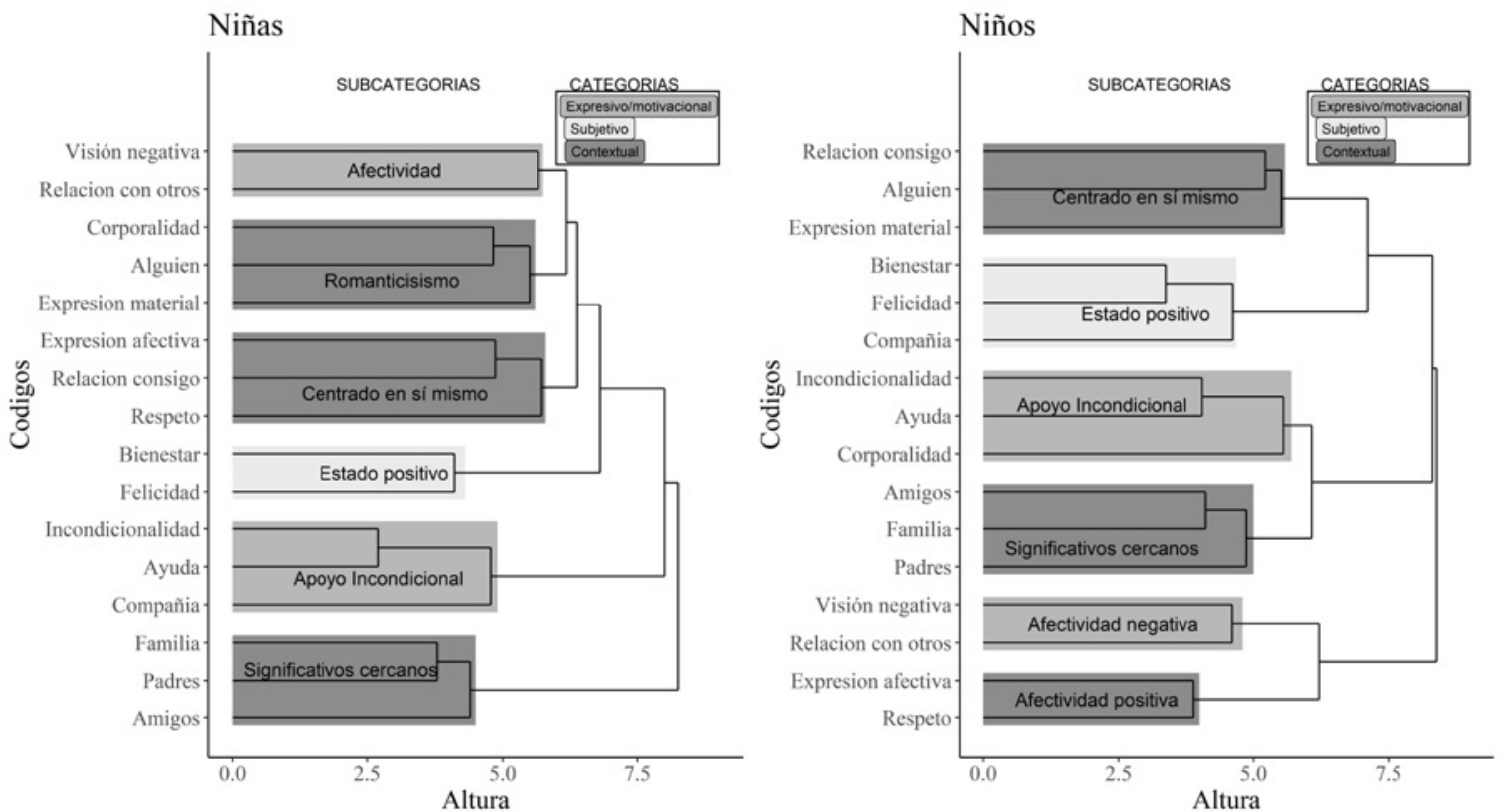
respecto al modelo general (véase Figura 3), no denotan tantas variaciones. Primero, es importante señalar que en el grupo de niños de 8 a 9 años los códigos compañía e incondicionalidad no aparecen en ninguno de los casos. En este mismo grupo, la subcategoría afectividad está conformada por visión negativa y relación con otros, mientras que en los niños y niñas de 10 a 12 años está compuesta por respeto, expresión afectiva y relación con otros. Asimismo, en este grupo la subcategoría romanticismo está conformada por el código expresión material. Una situación similar ocurre con la subcategoría apoyo incondicional, donde en los niños y niñas de 10 a 12 años emerge el código compañía, mientras que en los más pequeños no lo hace. Finalmente, la subcategoría centrado en sí mismo presenta variaciones entre los grupos, porque el código relación consigo mismo se asoció con diferentes códigos.

\section{Discusión}

El presente estudio tuvo como objetivo conocer, a través de su expresión escrita, el significado del amor en una muestra de niños y niñas de Lima Metropolitana, Perú. Los hallazgos señalan que al significado del amor infantil le subyacen seis subcategorías centrales: (a) significativos cercanos, (b) romanticismo, (c) centrado en sí mismo, (d) estado positivo, (e) afectividad y (f) apoyo incondicional. Un análisis cuidadoso de estas subcategorías conduce a pensar que el concepto de amor durante la niñez puede interpretarse desde tres grandes categorías: contextual o elicitador, experiencia subjetiva, y tendencia expresiva/ motivacional (Frijda et al., 2000; Haidt, 2003).

Con respecto a la categoría contextual, el amor es concebido por los niños como una emoción que se origina y expresa en un contexto de interacción significativa. En dicho contexto, cobra especial relevancia el vínculo que se produce con los padres y otros familiares, con los amigos, con otros pares en términos románticos, y con uno mismo. En este dominio se incluyen tres subcategorías: significativos cercanos, romanticismo y centrado en sí mismo. Estos hallazgos encuentran asidero en la teoría, ya que, conceptualmente, la palabra "amor" se ha utilizado para hacer referencia a una emoción que surge en un marco de proximidad y conexión interpersonal, y como algo que es valorado como seguro y positivo (Cavanaugh et al., 2015; Fredrickson, 2013).

De hecho, la literatura presenta sólidos argumentos teóricos para suponer que, en primera instancia, el amor en

Figura 3.

Dendrograma de los códigos, subcategorías y categorías según la edad
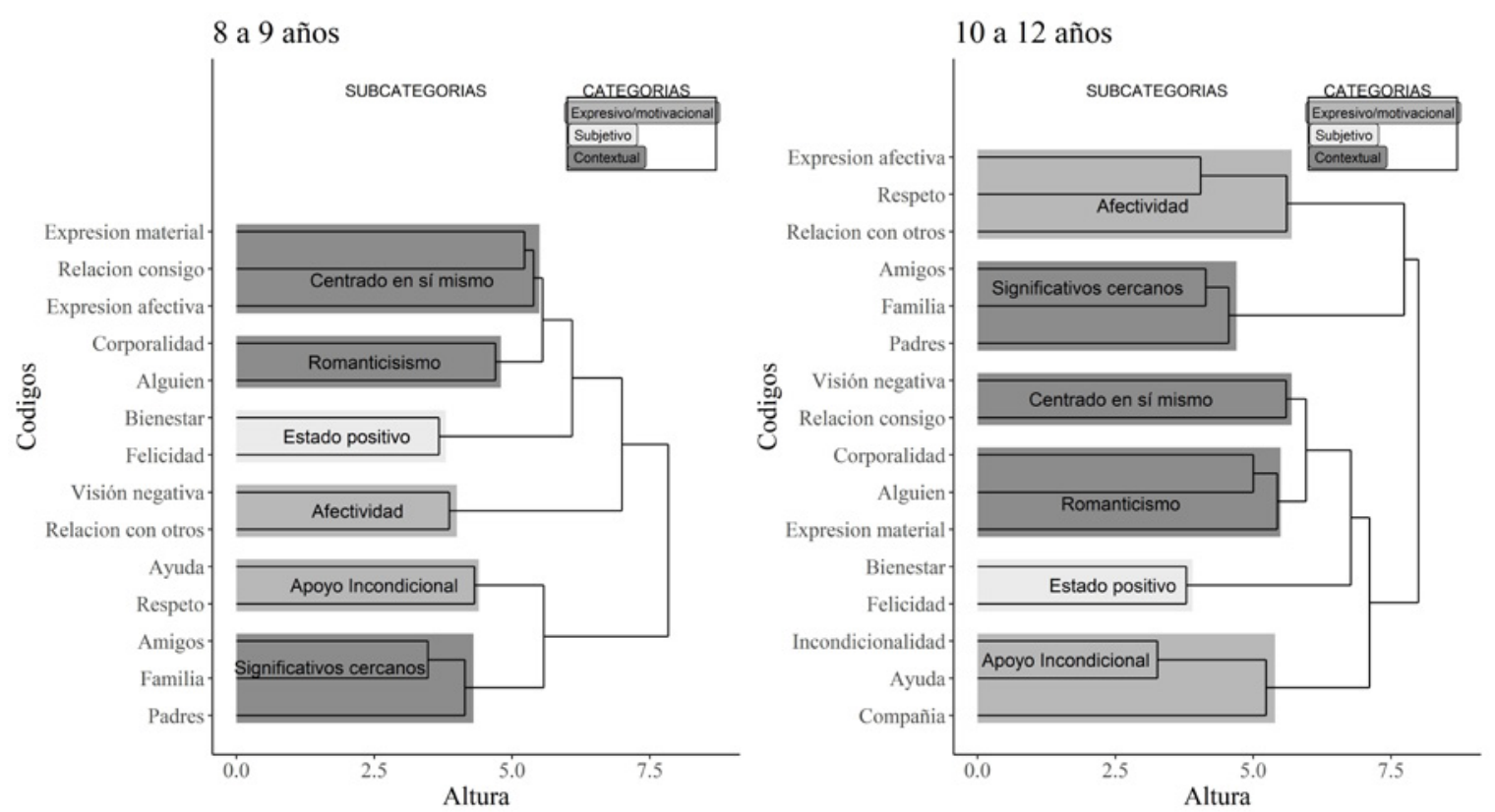
la etapa infantil se desarrolla en la interacción entre padres e hijos (Sabey et al., 2018). Los postulados clásicos sobre la teoría del apego (Bowlby, 1989) coinciden en afirmar que las primeras relaciones afectuosas con la figura de apego son sustanciales para el buen desarrollo del niño, e incluso para relaciones románticas en la etapa adulta (Sabelnikova et al., 2020). De ese modo, estos resultados señalan la importancia de construir lazos afectivos saludables entre padres e hijos (Melgosa \& Melgosa, 2006), no solo porque dicho vínculo resulta importante para el desarrollo de la salud psicológica (Phillips \& Shonkoff, 2000), sino porque es inherente a la definición misma del amor infantil. Por otro lado, se ha encontrado que familiares como los abuelos son vistos como modelos de identificación, figuras de sostén, cuidado, amor incondicional y fuente de consejos (Hess et al., 2020); y que los hermanos se ubican entre las personas más queridas, y son consideradas como fuente de compañía e intimidad (Facio \& Resett, 2007). Por ende, no resulta extraño que estas figuras integren el contexto interaccional que define la experiencia del amor durante la infancia.

Estos hallazgos son coherentes con algunos autores que argumentan que el amor en la etapa infantil se asocia con la amistad; en específico, con actividades como jugar o hacer algo juntos con otros compañeros (Grote \& Frieze, 1994; Klein, 1989). Aunque las relaciones con los padres tienen una preponderancia especial, las relaciones afectuosas con amigos también ocupan un lugar privilegiado, ya que estas dejan huellas duraderas en la formación del niño, por lo que resulta importante incrementar los estudios referidos a este tema (Mallet, 2016).

Dentro de esta misma categoría de análisis destaca también la subcategoría centrado en sí mismo, que resulta llamativa y digna de ser profundizada en futuros estudios. Más allá de eso, es interesante notar que, si bien fue en una proporción bastante ínfima, tanto en la muestra exploratoria como en la confirmatoria algunos niños se mencionaron como objeto de amor. Esto destaca la importancia que tiene el sentido de valoración personal durante la niñez, concepto que también resulta de gran importancia para el bienestar psicológico (Oros \& Richaud, 2011). Además, desde este punto, el amor está relacionado con aspectos socioculturales, debido a que en la actualidad - al menos desde la cultura occidental - existe un énfasis en el amor propio o autoestima como principio de desarrollo personal, el cual puede variar dependiendo del estrato socioeconómico donde viva el niño (Tabernero et al., 2017). Por otro lado, esta subcategoría contiene el código expresión material, que alude a que durante la niñez este tipo de amor se manifiesta a través de regalos o presentes, lo cual es usualmente observado en el contexto escolar como intercambio de cartas, dibujos, tarjetas o dulces. No obstante, es importante señalar el espacio sociohistórico en que se vive, pues los niños y niñas están expuestos a medios de comunicación — p. ej., redes sociales - que ejercen una fuerte influencia en la construcción del amor (Bonilla-Algovia \& Rivas-Rivero, 2020).

En cuanto al romanticismo, estos resultados guardan relación con diversos autores que señalan que el amor es un hecho romántico (Bartels \& Zeki, 2000; Cannoni \& Bombi, 2016; Rubin, 1970). Varios estudios coinciden en remarcar que durante la niñez media y tardía es posible identificar este tipo de amor, aunque no con la connotación que adquiere en la juventud o adultez. En algunos casos, los niños confunden amistad con romanticismo, y en general no pueden diferenciar claramente ambos tipos de vínculos, especialmente cuando se dan entre otras personas. En particular, el romanticismo está compuesto por el código de corporalidad, que alude a las manifestaciones fisiológicas típicas de este tipo de vinculación — real o fantaseada-, tales como aceleración del corazón, sensación de cosquilleos en el estómago, etc. Además, involucra el código alguien, lo que quiere decir que estas manifestaciones son sentidas hacia una persona no determinada; aspecto que lleva a pensar que el amor puede ser visto como un ente idealizado.

Respecto a la categoría subjetivo, los niños de esta muestra también han identificado al amor como una emoción de clara connotación o valencia positiva, que se experimenta como una sensación subjetiva de bienestar, contentamiento, alegría y felicidad. En este dominio se incluye la subcategoría estado positivo. Este resultado está en línea con los aportes de Fredrickson (2013), quien considera que el amor es una emoción de tono positivo por la sensación agradable que propicia y porque amplía el repertorio de pensamientos y acciones de las personas, construyendo recursos personales duraderos, en particular en torno a los vínculos sociales. De hecho, que los niños hayan asociado esta emoción con las experiencias particulares de bienestar, satisfacción, alegría y felicidad se encuentra respaldado por distintos investigadores, ya que, de acuerdo con estos, el amor desencadena, precisamente, emociones positivas 
que propician el deseo de explorar, jugar y disfrutar de los seres queridos (Brechet, 2015; Fredrickson, 2013).

Por otra parte, en cuanto a la categoría expresivo/motivacional, es posible advertir que para los niños de esta edad el amor es una emoción que moviliza conductas y actitudes benignas como respeto, expresiones amorosas, compañía, apoyo, consuelo y lealtad. En este dominio se incluyen las subcategorías afectividad y apoyo incondicional. Al respecto, se ha documentado que el amor motiva el cuidado del otro, pues las personas son movidas a enfocarse e invertir en el bienestar de otras personas, relegando momentáneamente cualquier tendencia a la autoabsorción (Fredrickson, 2013). En ese sentido, el amor en la etapa infantil es un asunto de afectividad que involucra aprecio, consideración y valoración al otro, que se expresa a través de abrazos, caricias, cariños y besos (Klein, 1989). De hecho, muchas veces este vínculo suele ser un apoyo incondicional a otra persona en momentos de dificultad, sin esperar recibir nada a cambio. Entonces, se trata de un asunto compasivo, porque se busca aliviar el dolor de otra persona, o remediarlo (Berscheid, 2010), comprometiéndose en situaciones difíciles (Grote $\&$ Frieze, 1994). De ahí que se suela asociar el amor a las conductas prosociales y altruistas (Cavanaugh et al., 2015).

Dentro de esta categoría, se conceptualiza al amor desde una visión negativa y que es exclusiva de los adultos — relación con el otro-, que está asociada a manifestaciones negativas como pérdida de tiempo, de mal gusto y rechazo a su existencia. Al respecto, Lazarus (2000) señala que el amor, en circunstancias desfavorables - - . ej., relación no correspondida, indiferencia-, a menudo viene acompañado de inseguridad, incertidumbre y angustia, y por eso es común que, cuando un niño se percibe como no amado, suele sentirse también triste y enojado (Widen \& Russell, 2005). Por ello, es posible que esta definición dada por los niños pueda estar influenciada por el contexto donde viven o su interacción con los adultos, puesto que el entorno familiar y social juega un papel importante en la percepción y conducta de sus integrantes (Thompson, 2008). Esto quiere decir que las manifestaciones de amor o desamor producidas en el hogar contribuyen en gran medida al significado del amor en la etapa infantil.

De otro lado, en relación con las diferencias del significado del amor según el sexo, se encuentran pequeñas diferencias en cuanto a su comparación, con la medida del efecto ( $h$ de Cohen). Sin embargo, respecto al contenido, se aprecia la no existencia de la subcategoría romanticismo en los niños, así como una fragmentación de la subcategoría afectividad, y que la compañía es un aspecto relacionado con el estado positivo en niños, y con el apoyo incondicional en niñas. Estos resultados revelan que el amor romántico es un asunto de mayor interés para las niñas; hallazgo que es coherente con postulados previos (Cannoni \& Bombi, 2016). Además, la afectividad puede tener variaciones cuando se considera una variable de comparación como el sexo (Brechet, 2015). Respecto a la subcategoría apoyo incondicional, la variación del código compañía podría revelar que puede ser valorado de forma diferente dependiendo del sexo, siendo entendido por los niños como expresión de un estado positivo, y para las niñas como apoyo incondicional. De hecho, se sabe teóricamente que en los niños el amor es sinónimo de amistad y de realizar actividades en conjunto (Klein, 1989), de ahí que la emoción positiva asociada a situaciones de juego pueda provocar estos cambios entre uno y otro clúster jerárquico.

En cuanto al significado del amor según la edad, los hallazgos muestran pequeñas diferencias si se considera la medida del efecto ( $h$ de Cohen). Sin embargo, un resultado interesante es la omisión de los códigos de compañía e incondicionalidad en los niños de 8 a 9 años, lo cual podría deberse a que la toma de consciencia de estos aspectos requiere mayores capacidades cognitivas — que de acuerdo con algunos estudios se alcanza entre los 10 a 12 años (van der Molen \& Molenaar, 1994) - o a que, mientras se cuenta con mayor edad, se tiene una preferencia de la compañía (Carlson \& Rose, 2007) y relaciones cercanas (Furman $\&$ Buhrmester, 1992). El hecho de que el código visión negativa se organice como una subcategoría de afectividad en niños pequeños podría indicar una relación no recíproca (Carlson \& Rose, 2007), porque algunos autores indican que el amor — al menos el romántico — presenta subidas y bajadas (Connolly et al., 2004). Pese a ello, todavía tiene sentido que las diferencias ocurridas puedan ser culturales.

Finalmente, es importante mencionar que estos resultados tienen tanto implicaciones teóricas, porque incrementan en modo sustancial la poca literatura acerca de la comprensión del amor en la etapa infantil en Hispanoamérica, como prácticas, porque permiten entender que el amor infantil está altamente relacionado con alguien cercano - como padres, familiares y amigos - y constituye uno de los principales motivos del desarrollo de la gratitud en la niñez (Oros et 
al., 2015). Teniendo esto en cuenta, la recomendación de un trato amable por parte de los padres o familiares resulta relevante, ya que, al no hacerlo, el niño se sentirá no amado; situación que impactará de forma negativa en la capacidad para modificar eventos problemáticos (McElwain et al., 2015), así como en el desarrollo de la habilidad para comprender las emociones de otros y responder a situaciones sociales de forma apropiada (Cooke et al., 2016), y porque podría ocasionar un déficit en el desarrollo de la empatía o establecimiento de relaciones más profundas (Sroufe, 2005), así como una predisposición a esperar que suceda lo peor (Zimmer-Gembeck et al., 2017).

Ahora bien, este estudio no está exento de limitaciones. Primero, en cuanto al tamaño muestral, se recomienda en futuros estudios incrementar la cantidad de participantes para examinar la estabilidad de los resultados. Segundo, es importante aumentar la cantidad de codificadores, pues en el presente estudio la codificación solo estuvo a cargo de dos investigadores - uno para la muestra exploratoria y otro para la versión confirmatoria—; por tanto, se recomienda contar con más codificadores por etapa de análisis, con el fin de llegar a acuerdos en caso de discrepancias. Tercero, aunque se analizaron las diferencias en el significado del amor según el sexo y la edad, sería prometedor en próximos estudios analizar posibles diferencias en cuanto a sus primeras relaciones de apego y otras conexiones sociales primarias. Y cuarto, se realizó la exploración del significado del amor mediante una pregunta genérica, pero sería interesante en futuros estudios explorar dichos significados mediante la escritura de un relato corto o un dibujo - que si bien se ha realizado previamente (Brechet, 2015), no existe evidencia científica en Latinoamérica- Esto permitiría explorar mayor cantidad de información respecto a un fenómeno tan complejo como el amor.

Como conclusión, se puede afirmar que el significado del amor en una muestra de niños y niñas peruanos puede ser interpretado desde las categorías de contexto elicitador, experiencia subjetiva y tendencia expresiva/motivacional; que, en cuanto al sexo de los participantes, las niñas demuestran mayor interés por los aspectos románticos del amor —además, el código compañía es percibido como un estado positivo para los niños y como apoyo incondicional para las niñas - ; y que, en cuanto a la edad, se evidenció la no presencia de los códigos de compañía e incondicionalidad en los niños de 8 a 9 años. Finalmente, se invita a continuar estudiando el fenómeno del amor en la niñez, especialmente en países hispanohablantes, debido a que la literatura reporta una escasez de este tipo de investigaciones.

\section{Referencias}

Adams, R. E., Laursen, B., \& Wilder, D. (2001). Characteristics of closeness in adolescent romantic relationships. Journal of Adolescence, 24(3), 353-363. https://doi.org/10.1006/jado.2000.0402

Anderson, E. L., Fraser, A., Caleyachetty, R., Hardy, R., Lawlor, D. A., \& Howe, L. D. (2018). Associations of adversity in childhood and risk factors for cardiovascular disease in mid-adulthood. Child Abuse \& Neglect, 76, 138-148. https://doi.org/10.1016/j.chiabu.2017.10.015

Aslanian, T. K. (2015). Getting behind discourses of love, care and maternalism in early childhood education. Contemporary Issues in Early Childhood, 16(2), 153165. https://doi.org/10.1177/1463949115585672

Bartels, A., \& Zeki, S. (2000). The neural basis of romantic love. Neuroreport, 11(17), 3829-3834. https://doi. org/10.1097/00001756-200011270-00046

Bernard, K., Hostinar, C. E., \& Dozier, M. (2019). Longitudinal associations between attachment quality in infancy, C-reactive protein in early childhood, and BMI in middle childhood: Preliminary evidence from a CPSreferred sample. Attachment \& Human Development, 21(1), 5-22. https://doi.org/10.1080/14616734.2018. 1541513

Berscheid, E. (2010). Love in the fourth dimension. Annual Review of Psychology, 61, 1-25. https://doi.org/10.1146/ annurev.psych.093008.100318

Bonilla-Algovia, E., \& Rivas-Rivero, E. (2020). Diseño y validación de la Escala de Mitos del Amor Romántico. Revista Iberoamericana de Diagnóstico y Evaluación-e Avaliação Psicológica, 4(57), 119-136. https://doi. org/10.21865/RIDEP57.4.09 
Bowlby, J. (1989). Una base segura. Paidós Ibérica.

Braun, V., \& Clarke, V. (2006). Using thematic analysis in psychology. Qualitative Research in Psychology, 3(2), 77-101. https://doi.org/10.1191/1478088706qp063oa

Brechet, C. (2015). Representation of romantic love in children's drawings: Age and gender differences. Social Development, 24(3), 640-658. https://doi.org/10.1111/ sode. 12113

Cannoni, E., \& Bombi, A. S. (2016). Friendship and Romantic Relationships During Early and Middle Childhood. SAGE Open, 6(3), 1-12. https://doi. org/10.1177/2158244016659904

Carlson, W., \& Rose, A. (2007). The Role of Reciprocity in Romantic Relationships in Middle Childhood and Early Adolescence. Merrill-Palmer Quarterly, 53(2). 262-290. https://doi.org/10.1353/mpq.2007.0008

Cavanaugh, L. A., Bettman, J. R., \& Luce, M. F. (2015). Feeling love and doing more for distant others: Specific positive emotions differentially affect prosocial consumption. Journal of Marketing Research, 52(5), 657673. https://doi.org/10.1509/jmr.10.0219

Chabot, S. (2008). Love and revolution. Critical Sociology, 34(6), 803-828. https://doi.org/10.1177/08969205080 95100

Cohen, J. (1988). Statistical power analysis for the behavioral sciences (2. ${ }^{\text {a }}$ ed.). Routledge.

Connolly, J., Craig, W., Goldberg, A., \& Pepler, D. (2004). Mixed-Gender Groups, Dating, and Romantic Relationships in Early Adolescence. Journal of Research on Adolescence, 14(2), 185-207. https://doi. org/10.1111/j.1532-7795.2004.01402003.x

Cooke, J. E., Stuart-Parrigon, K. L., Movahed-Abtahi, M., Koehn, A. J., \& Kerns, K. A. (2016). Children's emotion understanding and mother-child attachment: A meta-analysis. Emotion, 16(8), 1102-1106. https://doi. org/10.1037/emo0000221
Davis, C. G., Nolen-Hoeksema, S., \& Larson, J. (1998). Making sense of loss and benefiting from the experience: Two construals of meaning. Journal of Personality and Social Psychology, 75(2), 561-574. https://doi. org/10.1037/0022-3514.75.2.561

Facio, A., \& Resett, S. (2007). Desarrollo de las relaciones con padres y hermanos en adolescentes argentinos. Apuntes de psicología, 25(3), 255-266. http://www.apun tesdepsicologia.es/index.php/revista/article/view/78/80

Fredrickson, B. L. (2013). Positive Emotions Broaden and Build. Advances in Experimental Social Psychology, 47, 1-53. https://doi.org/10.1016/b978-0-12-407236-7. $00001-2$

Frijda, N. H., Manstead, A. S., \& Bem, S. E. (2000). Emotions and belief: How feelings influence thoughts. Cambridge University Press. https://doi.org/10.1017/ CBO9780511659904

Fromm, E. (2004). El arte de amar. Paidós.

Furman, W., \& Buhrmester, D. (1992). Age and Sex Differences in Perceptions of Networks of Personal Relationships. Child Development, 63(1), 103-115. https://doi.org/10.1111/j.1467-8624.1992.tb03599.x

Gan, G., Ma, C., \& Wu, J. (2007). Data clustering: theory, algorithms, and applications. ASA-SIAM Series on Statistics and Applied Probability. https://doi. org/10.1137/1.9780898718348

Grote, N. K., \& Frieze, I. H. (1994). The measurement of Friendship-based Love in intimate relationships. Personal Relationships, 1(3), 275-300. https://doi. org/10.1111/j.1475-6811.1994.tb00066.x

Haidt, J. (2003). The moral emotions. En R. J. Davidson, K. R. Scherer \& H. H. Goldsmith (eds.), Handbook of affective sciences (pp. 852-870). Oxford University Press.

Halfon, N., Forrest, C. B., Lerner, R. M., \& Faustman, E. M. (2018). Handbook of life course health development. Springer. 
Harlow, H. F. (1958). The nature of love. American psychologist, 13(12), 673-685. https://doi.org/10.1037/h00 47884

Harvey, M. W., Farrell, A. K., Imami, L., Carré, J. M., \& Slatcher, R. B. (2019). Maternal attachment avoidance is linked to youth diurnal cortisol slopes in children with asthma. Attachment \& Human Development, 21(1), 2337. https://doi.org/10.1080/14616734.2018.1541514

Hess, C. D., Schönfeld, F., Benítez, M., Zinoni, M., Rodriguez, L. M., \& Moreno, J. E. (2020). El vínculo entre abuelos y nietos: Conductas socio-educativas percibidas. Un estudio en la provincia de Entre Ríos. Revista Argentina de Ciencias del Comportamiento (RACC), 12(1), 221-222. https://revistas.unc.edu.ar/index.php/ racc/issue/view/2105/327

Johnson, R. B., \& Onwuegbuzie, A. J. (2004). Mixed Methods Research: A Research Paradigm Whose Time Has Come. Educational Researcher, 33(7), 14-26. https://doi.org/10.3102/0013189X033007014

Kassambara, A., \& Mundt, F. (2020). factoextra: Extract and Visualize the Results of Multivariate Data Analyses (1.0.7) [Computer software]. https://CRAN.R-project. org/package $=$ factoextra

Klein, P. S. (1989). Young children's understanding of love. International Journal of Early Childhood, 21(1), 27-34. https://doi.org/10.1007/bf03174571

Lazarus, R. S. (2000). Estrés y emoción. Manejo e implicaciones en nuestra salud. Desclée de Brouwer.

Lichtenthal, W. G., Currier, J. M., Neimeyer, R. A., \& Keesee, N. J. (2010). Sense and significance: A mixed methods examination of meaning making after the loss of one's child. Journal of Clinical Psychology, 66(7), 791-812. https://doi.org/10.1002/jclp.20700

Liu, X., Zhu, X. H., Qiu, P., \& Chen, W. (2012). A correlation-matrix-based hierarchical clustering method for functional connectivity analysis. Journal of Neuroscience
Methods, 211(1), 94-102. https://doi.org/10.1016/j. jneumeth.2012.08.016

Lucas, H., Csikszentmihalyi, M., \& Nakamura, J. (2019). Beyond-personal love-Experiencing love beyond the person. The Journal of Positive Psychology, 14(6), 789798. https://doi.org/10.1080/17439760.2019.1579354

Mallet, P. (2016). La amistad entre niños o adolescentes: Una fuerza que ayuda a crecer. Narcea Ediciones.

McElwain, N. L., Holland, A. S., Engle, J. M., Wong, M. S., \& Emery, H. T. (2015). Child-mother attachment security and child characteristics as joint contributors to young children's coping in a challenging situation. Infant and Child Development, 24, 414-434. https://doi. org/10.1002/icd.1886

Melgosa, J., \& Melgosa, A. D. (2006). Para La Pareja: Una relación estable para toda la vida. Editorial Safeliz.

Olabuénaga, J. I. R., \& Ispizua, M. A. (1989). La descodificación de la vida cotidiana: Métodos de investigación cualitativa. Universidad de Deusto.

Oros, L. B., \& Richaud, M. C. (2011). Cómo inspirar emociones positivas en los niños: Una guía para la escuela y la familia. Universidad Adventista del Plata.

Oros, L. B., Schulz-Begle, A., \& Vargas-Rubilar, J. (2015). Childrens Gratitude: Implication of Contextual and Demographic Variables in Argentina. Revista Latinoamericana de Ciencias Sociales, Niñez y Juventud, 13(1), 245-262. https://doi.org/10.11600/1692715x.1311 4130314

Papalia, D. E., \& Martorell, G. (2017). Desarrollo humano (13. ${ }^{\mathrm{a}}$ ed.). McGraw Hill.

Phillips, D. A., \& Shonkoff, J. P. (2000). From neurons to neighborhoods: The science of early childhood development. National Academies Press. https://doi.org/10.1722 6/9824 
Powell, H., Mihalas, S., Onwuegbuzie, A. J., Suldo, S., \& Daley, C. E. (2008). Mixed methods research in school psychology: A mixed methods investigation of trends in the literature. Psychology in the Schools, 45(4), 291-309. https://doi.org/10.1002/pits.20296

R Core Team. (2020). R: A language and environment for statistical computing (4.0.2) [Computer software]. https:/CRAN.R-project.org/

Rousseeuw, P. J. (1987). Silhouettes: A graphical aid to the interpretation and validation of cluster analysis. Journal of Computational and Applied Mathematics, 20, 53-65. https://doi.org/10.1016/0377-0427(87)90125-7

Rubin, Z. (1970). Measurement of romantic love. Journal of Personality and Social Psychology, 16(2), 265-273. https://doi.org/10.1037/h0029841

Sabelnikova, N., Kashirsky, D., \& Garvard, O. (2020). Attachment and Images of Parents and of the Romantic Partner of Russian Young Men and Women. Behavioral Sciences, 10(5), 87. https://doi.org/10.3390/bs 10050087

Sabey, A. K., Rauer, A. J., Haselschwerdt, M. L., \& Volling, B. (2018). Beyond "Lots of Hugs and Kisses": Expressions of Parental Love From Parents and Their Young Children in Two-Parent, Financially Stable Families. Family Process, 57(3), 737-751. https://doi. org/10.1111/famp.12327

Scott, M. (1997). La nueva psicología del amor. Emecé Editores.

Shaver, P. R., Morgan, H. J., \& Wu, S. (1996). Is love a "basic" emotion? Personal Relationships, 3(1), 81-96. https://doi.org/10.1111/j.1475-6811.1996.tb00105.x
Sroufe, L. A. (2005). Attachment and development: A prospective, longitudinal study from birth to adulthood. Attachment \& Human Development, 7(4), 349-367. https://doi.org/10.1080/14616730500365928

Tabernero, C., Serrano, A., \& Mérida-Serrano, R. (2017). Estudio comparativo de la autoestima en escolares de diferente nivel socioeconómico. Psicología Educativa, 23(1), 9-17. https://doi.org/10.1016/j.pse.2017.02.001

Thompson, R. A. (2008). Early attachment and later development: Familiar questions, new answers. En J. Cassidy \& P. R. Shaver (eds.), Handbook of attachment: Theory, research, and clinical applications (pp. 348-365). The Guilford Press.

Van der Molen, M. W., \& Molenaar, P. C. M. (1994). Cognitive psychophysiology: A window to cognitive development and brain maturation. En G. Dawson \& K. W. Fischer (eds.), Human Behavior and the Developing Brain (pp. 456-490). Guilford.

Widen, S. C., \& Russell, J. A. (2005). Do young children understand love as persisting? [Paper presentation]. En 17th Annual American Psychological Society Convention. Vienna, Austria.

Zimmer-Gembeck, M. J., Webb, H. J., Pepping, C. A., Swan, K., Merlo, O., Skinner, E. A., Avdagic, E., \& Dunbar, M. (2017). Review: Is parent-child attachment a correlate of children's emotion regulation and coping? International Journal of Behavioral Development, 41(1), 74-93. https://doi.org/10.1177/0165025415618276 